\title{
A Step-Down ZVS Power Converter with Self- Driven Synchronous Rectifier
}

\author{
${ }^{1}$ Najmehossadat Nourieh, ${ }^{2}$ Yichuang Sun and ${ }^{3}$ Oluyomi Simpson \\ School of Physics, Engineering and Computer Science (SPECS), University of Hertfordshire, \\ Hatfield, AL10 9AB, United Kingdom, \\ Email: 1n.nourieh@herts.ac.uk, ${ }^{2}$ y.sun@herts.ac.uk, ${ }^{3}$ o.simpson@herts.ac.uk
}

\begin{abstract}
In this paper a step-down ZVS power converter with a self-driven synchronous rectifier (SDSR) for a low-voltage high-current applications is proposed. A transformer leakage inductance, a resonant capacitor and a diode make up the active resonant network. To improve the performance of the converter, a SDSR with a center-tapped transformer is used at the secondary side of the converter. Consequently, due to transformer leakage inductance in secondary side, the output section requires no additional inductor, leading to a major size reduction of the circuit. For verification purposes, a laboratory prototype of the proposed converter is manufactured. Experimental results are presented for waveforms to validate the theoretical outcomes. Additionally, to substantiate the design of the proposed converter, a laboratory prototype is manufactured.
\end{abstract}

Keywords-Power converter, zero-voltage switching (ZVS), selfdriven synchronous rectification (SDSR).

\section{INTRODUCTION}

Improving power density and efficiency is essential for modern power supply. To reduce switching losses Softswitching techniques are developed. At zero-voltage switching (ZVS) conditions, not only the switch voltage-current overlap is eliminated, but also the turn-on losses created due to the MOSFET output capacitance $\left(\mathrm{C}_{\mathrm{oss}}\right)$ and reverse recovery of the $\mathrm{PN}$ junctions are removed properly [1]. In resonant converters, passive LC circuits are adopted to provide soft switching conditions. In ZVS resonant converters, the switching frequency is increased significantly while the switching, conducting and thermal losses are kept low suitably. Therefore, a ZVS resonant converter is one of the best candidates for a modern power supply [2].

Synchronous rectifiers (SR) present much lower conduction losses than the conventional $\mathrm{PN} /$ Schottky rectifying diodes [3]. The traditional methods use integrated circuits (IC) and self-driven synchronous rectifiers (SDSR). A major problem is the short-circuit condition occurred at the transition intervals created due to reverse-recovery of the PN junctions and the transformer secondary side leakage inductance [4]. In the SDSR, the gate signals are generated directly by the transformer auxiliary windings [5], [6]. Compared with gate driving methods which are using ICs, SDSR decreases the design complexity, production cost, elements number and gate driving loss [7], [8], [9]. A problem is the malfunction of SDSR in converters using dead time to regulate output voltage. This is because within dead-time intervals, the induced voltage on the auxiliary winding is reduced and consequently the SR may not be driven properly [10].

Integration of ZVS condition and SR is a challenging topic in many researches for different applications [11], [12].

This paper presents a novel converter which benefits from zero-voltage switching, resonance, isolation, integrated transformer and self-driven synchronous rectifier for lowvoltage high-current applications. Profiting from advantages of high efficiency, low switching, conducting and thermal losses, low EMI and gate driver loss, small size, high switching frequency range and isolation, this converter can be applied in various applications such as domestic electronic appliances, LED power sources, battery charging purposes, inductivelyheated appliances, wireless power charging of portable electronics, transcutaneous power transfer systems, biomedical implants, photovoltaic power optimizer, telecom and other centralized modular and distributed power applications [2], [7]. Furthermore, low-voltage high-current power electronic converters have received huge interest of both industry and academia for their application in stand-alone electric power generating systems.

The transformer leakage inductance (used as $\mathrm{L}_{\mathrm{r}}$ ) in conjunction with a resonant capacitor and a diode form an active resonant network (active resonant tank) showing a remarkable difference of the proposed converter from LLC [13]. Thereby, the transformer leakage inductance reflected on the transformer primary side is not problematic as it is used as resonant inductor. The employed active resonant tank decreases switching-frequency deviation over the whole range of load variations as well as provides ZVS operation for all switches [14], [15]. An inherent mechanism of this converter, due to its active resonant tank, drastically limits the switching frequency variations to appropriate small values (typically, $10 \%$ variations from zero-load to full-load). Thus, components can be designed optimally. A self-driven synchronous rectifier with center-tapped transformer is employed. The output section does not need any additional inductor; therefore, the converter size is greatly reduced.

The paper is organized as follows: Section II provides information about our proposed converter and its operational modes. Section III describes experimental results and finally, section IV concludes the paper.

\section{PROPOSED CONVERTER}

The proposed converter is presented in Fig. 1. The converter has seven operating modes in case of using diode rectifier [16] and ten modes in case of using SDSR shown in Fig. 2. The 
switches $Q_{1}$ and $Q_{2}$ constitute an inverter leg. The capacitors $C_{I}$ and $C_{2}$ (along with the MOSFET parasitic output capacitance $C_{\text {oss }}$ ) are set in parallel with the switches to provide ZVS condition at class D [1],[2]. An active resonant tank including $L_{r}, C_{r}$ and $D_{r}$ is employed. The transformer leakage inductance is designed to be used as resonant inductor $L_{r}$. The diode $D_{r}$ stabilizes the converter operation. Its junction capacitance is absorbed by $C_{r}$ and therefore its reverserecovery time is not problematic. The switches $S R_{I}$ and $S R_{2}$ are the output synchronous rectifiers driven by the transformer auxiliary windings. In Eq. (1),(2),(3) we define various parameters which will be used in the following discussion. The parameters $\alpha$ and $\beta$ are used to simplify the equations and $B$ is the converter normalized voltage gain " $B=n A=n V_{o} / V_{s}, \varphi_{1}$ and $\varphi_{2}$ are the conduction angles of $Q_{l}$ and $Q_{2}$ respectively. In each operation mode $\mathrm{I}_{\text {number }}=\mathrm{i}_{\mathrm{r}}\left(\mathrm{t}_{\text {number }}\right)$.

$$
\begin{aligned}
& \omega_{r}=2 \pi f_{r}=\frac{2 \pi}{T_{r}}=\frac{1}{\sqrt{L_{r} C_{r}}}, \quad R_{o}=\frac{n^{2} V_{o}{ }^{2}}{P_{\text {out }}} \\
& B=n \frac{V_{o}}{V_{S}}=n A \quad, \quad Z_{r}=\sqrt{\frac{L_{r}}{C_{r}}} \\
& \alpha=\frac{C_{r}}{C_{1}+C_{2}} \quad, \quad \beta=\frac{1+\alpha}{\alpha} \quad, \quad \omega_{\alpha}=\sqrt{1+\alpha} \omega_{r}
\end{aligned}
$$

Mode I $\left(\boldsymbol{t}_{0}<\boldsymbol{t}<\boldsymbol{t}_{1}\right)$ : prior to $t_{0}$, the diode $d_{l}$ was conducting and the gate signal for $Q_{l}$ has been set. Therefore, at $t_{0}$, the $Q_{l}$ is turned on under the ZVS condition. The voltage of the resonant capacitor (called resonant voltage $v_{r}$ ) is zero at $t_{0}$. Due to $L_{r}, v_{r}$ starts increasing in a resonant fashion when $Q_{l}$ conducts. Then, a positive voltage applies on the primary side of the transformer which reflects to the secondary and auxiliary windings (which provide gate voltage signal for $S R_{l}$ ). Then the switch $S R_{I}$ is turned on and power is delivered to the output.

$$
\begin{aligned}
& t_{1}-t_{0}=T_{1} \quad, \quad T_{1}=\frac{\varphi_{1}}{\omega_{r}} \\
& v_{r}(t)=V_{S}(1-B)\left[1-\cos \left(\omega_{r}\left(t-t_{0}\right)\right)\right] \\
& i_{r}(t)=\frac{V_{S}}{Z_{r}}\left[(1-B) \sin \left(\omega_{r}\left(t-t_{0}\right)\right)\right]
\end{aligned}
$$

Mode II $\left(\boldsymbol{t}_{1}<\boldsymbol{t}<\boldsymbol{t}_{2}\right)$ : by reaching $v_{r}$ to $V_{S}$ the voltage across the primary winding is reduced. Therefore, the auxiliary voltage applied on the gate of $S R_{1}$ is also decreased to less than the threshold voltage which causes the MOSFET turned off. However, due to the transformer leakage inductance at the secondary side, $L_{S S 2}$, the anti-parallel diode of $S R_{I}$ remains ON and continues the current.

At the end of this mode, $Q_{l}$ is turned off. Due to $C_{l}, Q_{l}$ is turned off at ZVS condition. The durations of this mode along with the previous mode, in which $Q_{l}$ is conducting, is defined as $\varphi_{1} / \omega_{r}$. To provide the phase lag needed to maintain ZVS turn-off, $i_{r}$ should be positive at $t_{2}$, or in other words $\varphi_{1}<180$. All equations of this mode are identical to those of the previous interval.

Mode III $\left(\boldsymbol{t}_{2}<\boldsymbol{t}<\boldsymbol{t}_{3}\right)$ : since $t_{2}$ is prior to the zero-crossing instant of the resonant current, $i_{r}$; by turning $Q_{1}$ off at $t_{2}$, the remaining current of $L_{r}$ flows through $C_{1}$ and $C_{2}$. Then, the middle voltage (the voltage of node $M$ in Fig. 1 which is denoted by $v_{M}$ ), is reduced to zero through a resonant with $L_{r}$ at $t_{3}$.

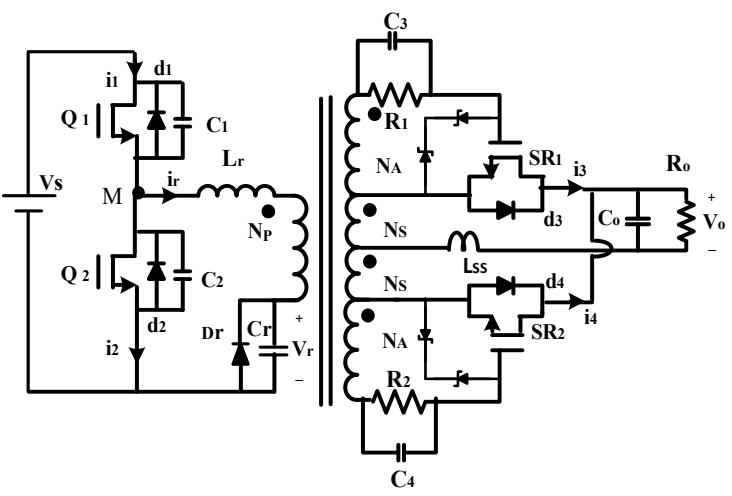

Fig. 1. Proposed resonant ZVS converter

$\frac{i_{r}(t)}{V_{s} / Z_{r}}=\frac{(1-B) \cos \varphi_{1}}{\sqrt{1+\alpha}} \sin \left(\omega_{\alpha}\left(t-t_{1}\right)\right)+$

$(1-B) \sin \varphi_{1} \cos \left(\omega_{\alpha}\left(t-t_{1}\right)\right)$

$\frac{v_{r}(t)}{V_{s}}=\frac{(1-B) \cos \varphi_{1}}{\sqrt{1+\alpha}} \sin \left(\omega_{\alpha}\left(t-t_{1}\right)\right)-$

$\frac{(1-B) \sin \varphi_{1}}{1+\alpha} \cos \left(\omega_{\alpha}\left(t-t_{1}\right)\right)$

Mode IV $\left(\boldsymbol{t}_{3}<\boldsymbol{t}<\boldsymbol{t}_{4}\right)$ : at $\mathrm{t}_{3}$, the diode $d_{2}$ is forward biased and $i_{r}$ flows through it until $t_{4}$, when the resonant current $i_{r}$ becomes zero. The auxiliary winding applies a positive voltage on the gate of $S R_{2}$ and turns it on. In this mode, the gate signal for $Q_{2}$ is set to turn it on at ZVS. At $t_{3}$ the current of $I_{r}$ is called $I_{3}$ which is used in Eq. (10). Here, $\varphi\left(\mathrm{t}_{4}<\mathrm{t}<\mathrm{t}_{5}\right)$ and $\varphi\left(\mathrm{t}_{5}<\mathrm{t}<\mathrm{t}_{6}\right)$ are called $\varphi_{\mathrm{x} 1}$ and $\varphi_{\mathrm{x} 2}$. Considering $\frac{-B}{\sin \varphi_{2}}=\gamma$ the equations are as below.

$\frac{i_{r}(t)}{V_{s} / Z_{r}}=(2 B-\gamma) \sin \left(\omega_{r}\left(t-t_{3}\right)\right)$

$\frac{v_{r}(t)}{V_{S}}=V_{s}\left[I_{3} \sin \left(\omega_{r}\left(t-t_{3}\right)\right)+B\right]$

$\varphi_{x 1}+\varphi_{x_{2}}=\varphi_{d}$

Mode $\boldsymbol{V}\left(\boldsymbol{t}_{4}<\boldsymbol{t}<\boldsymbol{t}_{5}\right)$ : at $\mathrm{t}_{4}$ the current of $Q_{2}$ becomes positive and then the MOSFET of $Q_{2}$ conducts. Due to the transformer secondary side leakage inductance $L_{S S 2}$, the diode $\mathrm{d}_{3}$ remains ON until $\mathrm{t}_{5}$, when the current of $L_{S S 2}$ is reversed and $d_{3}$ is turned OFF at ZCS condition. During this mode, the energy stored in $C_{r}$ is transferred to the output, but $v_{r}$ is still positive at $t_{5}$.

$\frac{i_{r}(t)}{V_{s} / Z_{r}}=(2 B-\gamma) \sin \left(\omega_{r}(t-t 4)\right)$ 


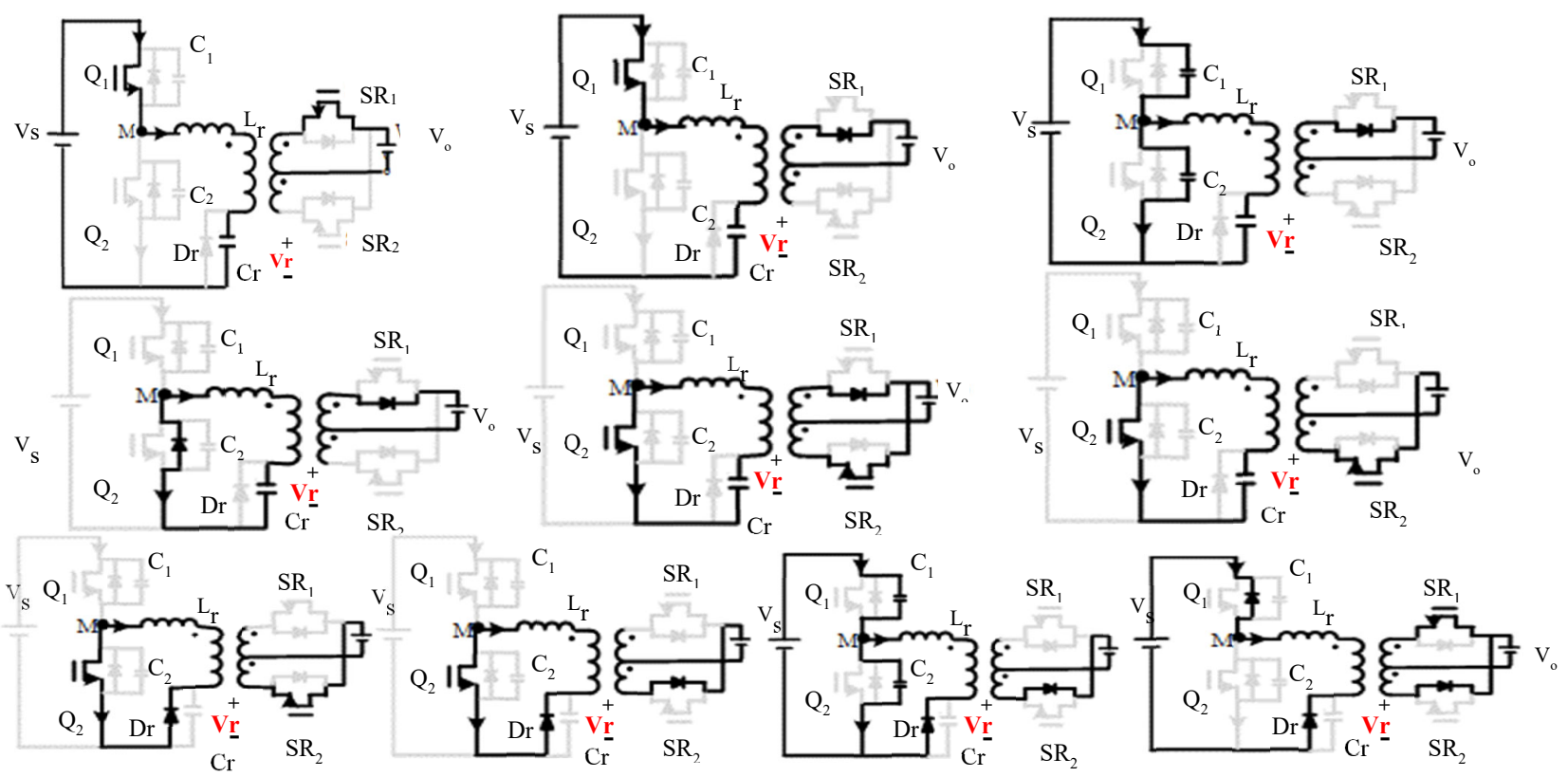

Fig. 2. Schematic circuit of the proposed high efficiency isolated resonant converter using a self-driven synchronous rectifier

$$
\frac{v_{r}(t)}{V_{S}}=-(2 B-\gamma) \cos \left(\omega_{r}\left(t-t_{4}\right)\right)+B
$$

Mode VI $\left(t_{5}<t<t_{6}\right)$ : during this mode, remaining energy stored in $C_{r}$ is transferred to the output until $v_{r}$ reaches zero at $t_{6}$. The interval from $t_{4}$ to $t_{6}$ is defined as $\varphi_{d}$ which indicates the conduction angle of $D_{r}$.

$$
\begin{aligned}
& \frac{v_{r}(t)}{V_{S}}=(2 B-\gamma)\left[\frac{\left(\cos \varphi_{x 2}\right)}{\left(\sin \varphi_{x 1}\right)} \sin \left(\omega_{r}(t-t 5)\right)\right. \\
& \left.+\cos \left(\omega_{r}(t-t 5)\right)\right] \\
& \frac{i_{r}(t)}{V_{s} / Z_{r}}=(2 B-\gamma)\left[-\frac{\left(\cos \varphi_{x 2}\right)}{\left(\sin \varphi_{x 1}\right)} \cos \left(\omega_{r}(t-t 5)\right)\right. \\
& \left.\left.+\cos \left(\omega_{r}(t-t 5)\right)\right]+\frac{\left(\sin \varphi_{x 2} \cot \varphi_{x 1}\right)}{C_{r}}+\frac{\cos \varphi_{x 2}}{C_{r}}\right] \\
& T_{5}+T_{6}=\frac{\varphi_{d}}{\omega_{r}}, \quad \varphi_{d}=\cos ^{-1}\left(\frac{B}{2 B-\gamma}\right)
\end{aligned}
$$

Mode VII $\left(\boldsymbol{t}_{6}<\boldsymbol{t}<\boldsymbol{t}_{7}\right)$ : by setting $v_{r}$ to zero at $t_{6}$, the diode $D_{r}$ is forward biased at ZVS. Then the resonant current $i_{r}$ continues through it and the voltage of $v_{r}$ is clamped at zero. During this mode, the magnetic energy stored in $L_{r}$ is transferred to the output and the magnitude of $i_{r}$ decreases linearly.

$$
\frac{i_{r}(t)}{V_{s} / Z_{r}}=B \omega_{r}\left(t-t_{6}\right)+r_{r}\left(t_{6}\right)
$$

Mode VIII $\left(t_{7}<t<t s\right)$ : at $\mathrm{t} 7$ the voltage of auxiliary winding of SR2 is reduced to less than the threshold voltage. Hence, SR2 is turned off while its anti-parallel diode $\mathrm{d} 3$ is still conducting. The preceding equations are remained valid for this period, since the substitution of SR2 with its anti- parallel diode applies no changes.

$T_{x}=T_{7}+T_{8}=\frac{\varphi_{3}}{\omega_{r}}$

(19) $i_{x}=i_{7}+i_{8}$

$\frac{v_{r}(t)}{V_{S}}=V_{s}\left[\left(-I_{x} \sqrt{\alpha}\right) \sin \left(\omega_{r} \sqrt{\alpha}\left(t-t_{x}\right)\right)\right.$

$\left.+B \cos \left(\omega_{r} \sqrt{\alpha}\left(t-t_{x}\right)\right)-B\right]$

$\frac{i_{r}(t)}{V_{s} / Z_{r}}=\left[\frac{B}{\sqrt{\alpha}} \sin \left(\omega_{r} \sqrt{\alpha}\left(t-t_{x}\right)\right)\right.$

$\left.+I_{x} \cos \left(\omega_{r} \sqrt{\alpha}\left(t-t_{x}\right)\right)\right]$

Mode IX $\left(t_{8}<t<t 9\right)$ : by turning $Q_{2}$ off at $\mathrm{t}_{8}$, the flow of the remaining current in $L_{r}$ continues through $C_{1}$ and $C_{2}$ resulting that $v_{M}$ increases until it reaches $V_{S}$ at $\mathrm{t}_{9}$.

$\frac{i_{r}(t)}{V_{s} / Z_{r}}=\omega_{r}\left(t-t_{9}\right)+i_{r}\left(t_{9}\right)$

$t_{10}-t_{9}=\frac{i_{r}\left(t_{9}\right)}{\omega_{r}}$

Mode $\boldsymbol{X}\left(\boldsymbol{t}_{9}<\boldsymbol{t}<\boldsymbol{t}_{10}\right)$ : by reaching $v_{M}$ to $V_{S}$ at $t_{9}$, the diode $d_{l}$ is turned on at ZVS and $i_{r}$ flows through it until it becomes zero at $t_{10}$. Then, $d_{1}$ and $D_{r}$ are turned off at ZCS conditions. It should be mentioned here that no additional mode will be added by $C_{3}$ and $C_{4}$. 

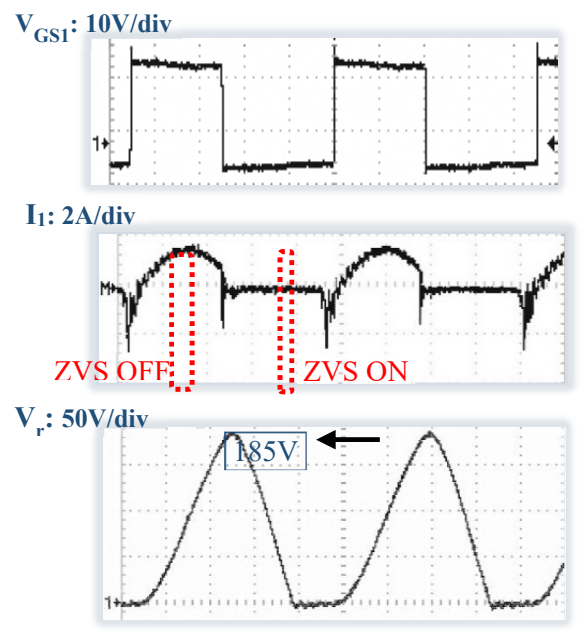

$\mathrm{V}_{\mathrm{GS} 2}: 10 \mathrm{~V} / \mathrm{div}$

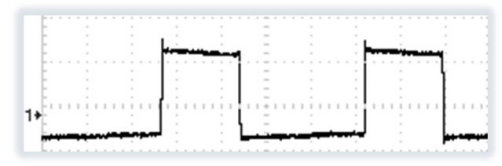

$\mathrm{I}_{2}: 2 \mathrm{~A} / \mathrm{div}$

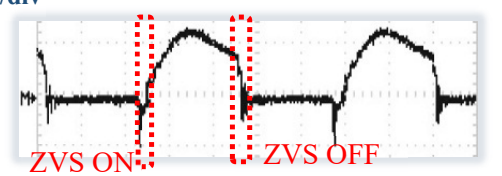

$\mathrm{V}_{\mathrm{DS}(\mathrm{SR} 1)}: 10 \mathrm{~V} / \mathrm{div}$

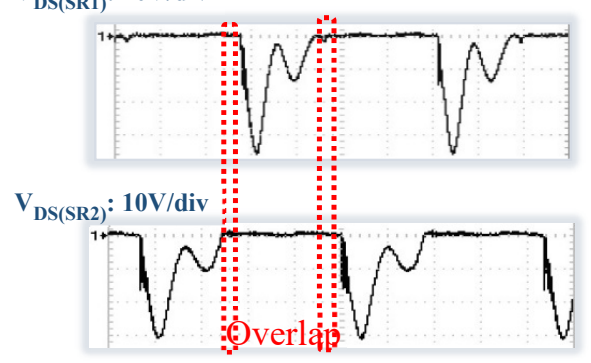

Fig. 3 Practical waveforms fo the converter in full load mode $($ Pout $=100 \mathrm{~W}, \mathrm{Fs}=220 \mathrm{kHz}, 1 \mathrm{us} / \mathrm{div}$, Period=4.54 us)

\section{EXPERIMENTAL RESULTS}

A prototype of the proposed converter has been made in laboratory with specifications such as: input voltage $V_{s}=150 \mathrm{~V}$, output voltage $V_{o}=5 \mathrm{~V}$, output current $I_{o}=20 \mathrm{~A}$, switching frequency $F_{s-\text { full }}=220 \mathrm{kHz}$ for full-load waveforms. The parameters of this converter is given in table I (overdesign $=1.2, \quad \alpha=6$ (obtained by the simulation in PSPICE)).

Also, the efficiency of this converter is $94.5 \%$. Figure. 3 shows practical waveforms for primary side of transformer in fullload case, where both "ZVS turn-on" and "ZVS turn-off" are obtained for $Q_{1}$ and $Q_{l}$. In addition, the currents of $Q_{1}$ and $Q_{1}$ are negative for a few tenths of microseconds, when the primary side switches are turned on and off. The behavior of $\mathrm{V}_{\mathrm{r}}$ is also shown in this figure. Then the experimental waveforms of secondary side are illustrated, where two drain- source voltages of SRs with mentioned overlapping periods are highlighted.

TABLE I

PARAMETERS AND COMPONENTS OF THE CIRCU
\begin{tabular}{|c|c|}
\hline Symbol & Full-Load - Light-load \\
\hline $\mathrm{Vs}_{\mathrm{s}}$ & $150 \mathrm{~V}$ \\
\hline $\mathrm{Vo}_{\mathrm{o}}$ & $5 \mathrm{~V}$ \\
\hline $\mathrm{P}_{\text {out }}$ & $100 \mathrm{~W}$ \\
\hline $\mathrm{f}_{\mathrm{s}}$ & $220 \mathrm{kHz}$ \\
\hline $\mathrm{C}_{\mathrm{r}}$ & $23 \mathrm{nF}$ \\
\hline $\mathrm{L}_{\mathrm{r}}$ & $25 \mu \mathrm{H}$ \\
\hline $\mathrm{C}_{1}$ & $1 \mathrm{nF}$ \\
\hline $\mathrm{C}_{2}$ & $1 \mathrm{nF}$ \\
\hline $\mathrm{R}_{1}$ & $0.5 \Omega$ \\
\hline $\mathrm{R}_{2}$ & $0.5 \Omega$ \\
\hline $\mathrm{f}_{\mathrm{r}}$ & $202 \mathrm{kHz}$ \\
\hline
\end{tabular}

\section{CONCLUSION}

Working under "Zero Voltage Switching (ZVS)" for lowvoltage and high-current applications, a new isolated resonant converter with SDSR is presented. The active resonant network is composed of a transformer leakage inductance, a resonant capacitor and a diode which provide ZVS conditions for all switches. A simple SDSR with a center-tapped transformer is employed at the secondary side to promote converter performance and three transformers are merged into one. Hence the output section needs no further inductor, resulting in a major size reduction of the circuit. Experimental results are presented for waveforms to validate the theoretical outcomes.

\section{REFERENCES}

[1] M. Jabbari, "Unified Analysis of Switched-Resonator Converters", IEEE Trans. on Power Electronics, Vol. 26, No. 5, 2011.

[2] A. Ayachit, F. Corti, A. Reatti and M. Kazimierczuk, "Zero-Voltage Switching Operation of Transformer Class-E Inverter at Any Coupling Coefficient", IEEE Trans. On Industrial Electronics, Vol. 66, No. 3, 2019.

[3] K. Jin, L. Gue, and J.Wang, "A 10-MHz Resonant Converter With a Synchronous Rectifier for Low-Voltage Applications", IEEE Trans. on Power Electronics., Vol. 34, No. 4, 2019.

[4] W. X. Zhong, S. Y. Hui, W. C. Ho and X. Liu, "Using Self Driven AC-DC Synchronous Rectifier as a Direct Replacement for Traditional Power Diode Rectifier", IEEE Trans Industrial Electronics, Vol. 59, No. $1,2012$.

[5] C. Liu ; D. Mandal ; Z. Yao ; M. Sun ; J. Todsen; B. Johnson ; S. Kiaei; B. Bakkaloglu, "A 50-V Isolation, 100-MHz, 50-mW SingleChip Junction Isolated DC-DC Converter With Self-Tuned Maximum Power Transfer Frequency", IEEE Trans on Circuits and Systems II, Vol. 66, No. 6, 2019.

[6] D. Rothmund, T. Guillod, D. Bortis, "99.1\% Efficient 10 kV SiC-Based Medium-Voltage ZVS Bidirectional Single-Phase PFC AC/DC Stage", IEEE Journal of Emerging and Selected Topics in Power Electronics, Vol. 7, No. 2, 2019.

[7] C. Duan, K. Bai, W. Guo and Z. Nie, "Design of A $2.5 \mathrm{~kW} 400 \mathrm{~V} / 12 \mathrm{~V}$ High-Efficiency DC/DC Converter Using A Novel Synchronous Rectification Control for Electric Vehicles", IEEE Trans. on Transportation Electrification, Vol. 1, No.1, 2015. 
[8] K. Kim, J. Kwon, H. Lee and B. Kwon, "Single-stage High-Power Factor Half-Bridge Flyback Converter With Synchronous Rectifier", IET Power electronics, Vol. 7, No. 1, 2014.

[9] M .J. Scott, K. Zou, J. Wang, C. Chen, M. Su and L. Chen, "A Gallium Nitride Switched-Capacitor Circuit Using Synchronous Rectifier", IEEE Trans. on Industry Applications, Vol. 49, No. 3, 2013.

[10] K. Jin, Z. Liu, X. Yu and X. Ren, "A Self-Driven Current-DoublerRectifier Three-Level Converter with Integrated Magnetics", IEEE Trans. on Power Electronics, Vol. 29, No. 1, 2014.

[11] G. Chen, Y. Deng, Y. Tao, X. He, Y. Wang and Y. Hu, "Topology Derivation and Generalized Analysis of Zero-Voltage-Switching Synchronous DC-DC Converters with Coupled Inductors", IEEE Trans. on Industrial Electronics, Vol. 63, No. 8, 2016.

[12] N. Z. Yahaya, K.M. Begam and M. Awan, "Experimental Analysis of anew Zero-Voltage Switching Synchronous Rectifier Buck converter", IET Power Electronics, Vol. 4, No. 7, 2011.

[13] P. He and A. Khaligh, "Comprehensive Analyses and Comparison of $1 \mathrm{~kW}$ Isolated DC-DC Converters for Bidirectional EV Charging Systems", IEEE Trans. on Transportation Electrification, Vol. 3, No. 1, 2017.

[14] K. Jong-Woo and A. Gun-Woo, "A New LLC Series Resonant Converter with a Narrow Switching Frequency Variation and Reduced Conduction losses", IEEE Trans. on Power Electrons. Vol. 29, No. 8, 2011.

[15] E. Asa, K. Colak and D. Czarkowski, "Analysis of CLL Resonant Converter with Semi Bridgeless Active Rectifier and Hybrid Control", IEEE Trans. Industrial Electronics, Vol. 62, No. 11, 2015.

[16] A. Mohtasham and M. Jabbari, "Design and Simulation of a High Frequency Isolated Converter with Zero Voltage Switching", Master thesis, Islamic Azad University of Najafabad, Isfahan, Iran, Spring 2014. 